\title{
Homofobia Transatlântica: Preconceito contra Lésbicas e Gays em Portugal e no Brasil
}

\author{
Jorge Gato ${ }^{1}$ \\ Anne Marie Fontaine \\ Centro de Psicologia da Universidade do Porto, Porto, Portugal \\ Vanessa Barbosa Romera Leme \\ Programa de Pós-Graduação em Psicologia da Universidade Salgado de Oliveira- \\ Universo, Niterói, RJ, Brasil \\ Alessandro André Leme \\ Programa de Pós-Graduação em Sociologia da Universidade Federal Fluminense, \\ Niterói, RJ, Brasil
}

\begin{abstract}
Resumo
Neste estudo efetuou-se uma comparação do preconceito contra lésbicas e gays em Portugal e no Brasil, numa amostra de estudantes universitários $(N=844)$. Foram avaliadas duas manifestações do preconceito: uma de caráter mais tradicional (homopatologização) e outra de caráter mais contemporâneo (heterossexismo moderno). Uma vez que este tipo de preconceito não é independente do gênero, foi também explorada a relação com essa variável. Não se verificaram diferenças entre os dois países, quer no que diz respeito aos níveis de homopatologização, quer de heterossexismo moderno. Os participantes portugueses e brasileiros evidenciaram níveis significativamente mais elevados de heterossexismo moderno do que de homopatologização. No que diz respeito ao gênero, os homens evidenciaram níveis mais elevados dos dois tipos de preconceito do que as mulheres. Os resultados deste estudo chamam a atenção para a necessidade de programas de luta contra a homofobia. Os agentes educativos e psicossociais, particularmente aqueles que operam no espaço universitário, devem ser sensíveis, quer a expressões mais modernas do preconceito, quer à maior incidência deste na população masculina.
\end{abstract}

Palavras-chave: Homofobia, preconceito tradicional, preconceito moderno, gênero.

\section{Transatlantic Homophobia: Prejudice against Lesbians and Gay Men in Portugal and Brazil}

\begin{abstract}
In this study we compared prejudice against lesbians and gay men in Portugal and Brazil, in a sample of university students $(N=844)$. Two forms of prejudice were assessed: one with a more traditional nature (pathologization of homosexuality) and a more contemporary one (modern heterosexism). Because these prejudices are not independent of gender, we explored the relation with this variable. No differences
\end{abstract}

Enderço para correspondência: Universidade do Porto Faculdade de Psicologia e de Ciências da Educação, Rua Alfredo Allen, 4200-135, Porto, Portugal, E-mail: jorgegato@fpce.up.pt

Este trabalho foi financiado por uma bolsa de doutoramento atribuída pela Fundação para a Ciência e Tecnologia (SFRH/BD/41752/2007).

Este trabalho foi anteriormente publicado na revista Passages de Paris e o autor obteve autorização do corpo editorial da mesma, para submissão a revista científica indexada e com revisão por pares. 
were found between the two countries in both variables. Portuguese and Brazilian participants evidenced significantly higher levels of modern heterosexism than of pathologization of homosexuality. As far as the gender of participants is concerned, men scored higher than their female counterparts in both types of prejudice. Results of this study draw attention to the need of homophobia reducing interventions. Educational and psychosocial agents, particularly those who work in university environments should be especially attentive both to modern expressions of prejudice against lesbians and gay men, and to its major prevalence in the male population.

Keywords: Homophobia, traditional prejudice, modern prejudice, gender.

\section{Homofobia Transatlántica: Prejuicio Contra Lesbianas y Gays en Portugal y Brasil}

\section{Resumen}

En este estudio se realizó una comparación del prejuicio contra lesbianas y gays en Portugal y Brasil, en una muestra de estudiantes universitarios $(N=844)$. Se evaluaron dos manifestaciones de prejuicio: una más tradicional (homopatologización) y otra de carácter más actual (heterosexismo moderno). Dado que este tipo de prejucio no es independiente del género, también se exploró la relación con esta variable. No hubo diferencias entre ambos países, con respecto a los niveles de homopatologización y los de heterosexismo moderno. Los participantes portugueses y brasileños mostraron niveles significativamente más altos de Heterosexismo moderno que de Homopatologización. Con respecto al género, los varones mostraron niveles más altos de los dos tipos de prejucios que las de mujeres. Los resultados de este estudio señalan la necesidad de programas de lucha contra la homofobia. Los agentes educativos y psicosociales, en particular los que actúan en el espacio universitario, deben ser sensibles a las expresiones más modernas de prejuicio, ya que la mayor incidencia se encuentra en la población masculina.

Palabras clave: Homofobia, prejuicios tradicionales, prejuicio moderno, género.

Existe uma grande variabilidade a nível mundial no que diz respeito ao preconceito e discriminação contra lésbicas e gays (Jackle \& Wenzelburger, 2014; Stulhofer \& Rimac, 2009; Takács \& Szalma, 2013; van den Akker, van der Ploeg, \& Scheepers, 2013). Porém, dados provenientes dos dois momentos de coleta mais recentes do World Values Survey - WVS (1999-2004, 2005-2009), revelam índices semelhantes de preconceito contra esta população no Brasil e em Portugal. Por exemplo, quando se pergunta em que medida a homossexualidade é justificável (10 = nada justificável), as médias são respectivamente para Brasil e Portugal de 6,72 e 7,64 (Jackle \& Wenzelburger, 2014). Partilhando a mesma língua e uma longa história, é esperado que as atitudes sociais referentes a esta temática sejam comparáveis nos dois países. Contudo, indo um pouco para além dos indicadores socio- lógicos, como o referido anteriormente, é importante compreender as diversas nuances, em termos psicológicos, que o preconceito contra lésbicas e gays poderá assumir em Portugal e no Brasil.

Em 1954, Allport definiu preconceito como, "uma atitude aversiva ou hostil relativamente a uma pessoa que pertence a um grupo, pelo simples fato de pertencer a esse grupo, presumindo-se, portanto, que possui as características censuráveis atribuídas ao grupo" (1954/1997, p. 7). Embora este constructo tenha sido entendido de diferentes maneiras ao longo do tempo, é consensualmente aceito que resulta de uma antipatia, ou expressão de emoções negativas em relação a um determinado grupo associada a um estereótipo ou pré-julgamento acerca de um grupo ou dos seus membros (Plous, 2003; Quillian, 2006). De acordo com uma categoriza- 
ção clássica das atitudes, os estereótipos corresponderiam assim ao componente mais cognitivo das atitudes, o preconceito ao componente mais afetivo e a discriminação ao componente mais comportamental (Fiske, 1998). Presentemente, o preconceito é definido, de uma forma mais lata, como uma avaliação negativa (i.e., uma atitude) que se refere a um grupo ou a um indivíduo com base na sua pertença a um grupo (Dovidio \& Gaertner, 2010; Duckitt, 1992).

Herek (2009) utiliza o termo preconceito sexual para se referir a uma atitude negativa face a um indivíduo tendo por base a sua pertença a um grupo definido em termos das suas atrações sexuais, comportamentos ou orientação. Sendo uma atitude, o preconceito sexual baseia-se em informação que os indivíduos retiram das suas respostas afetivas, crenças e comportamentos (Herek, 2009). Segundo o autor, a sua mensuração implica inevitavelmente referenciar a orientação sexual, um fenômeno multidimensional que compreende muitos componentes das relações íntimas, incluindo o desejo erótico, a atração romântica, e o comportamento sexual. A orientação sexual remete também para uma identidade pessoal e coletiva, e para leis e políticas que estão frequentemente no centro de disputas políticas, religiosas e morais (Herek \& McLemore, 2013). Perante esta complexidade, as definições operacionais de preconceito sexual podem-se focar em diferentes fenômenos, incluindo atitudes face à atração entre pessoas do mesmo sexo ou comportamentos sexuais; atitudes face à "homossexualidade" ou "bissexualidade" genericamente consideradas; atitudes face a lésbicas, gays, mulheres bissexuais ou homens bissexuais; e atitudes face a aspectos institucionais do estigma sexual, como por exemplo leis que dizem respeito ao casamento e à parentalidade (Herek \& McLemore, 2013).

Já o conceito de homofobia tem sido utilizado para descrever as atitudes e os comportamentos anti-homossexuais de carácter individual (Weinberg, 1972). Sendo uma designação bastante contestada, nomeadamente pela ausência de um carácter fóbico e por remeter este tipo de discriminação para um plano puramente individual (Gato, Carneiro, \& Fontaine, 2011), a homofobia é ainda o termo mais amplamente difundido (Hegarty, 2006; Nardi, 2013). Nesta medida, os termos "atitudes negativas", "preconceito sexual" e "homofobia" são utilizados de forma indistinta neste trabalho.

O desenvolvimento de um pretenso novo sentido moral de igualdade, respeito e tolerância nas sociedades ditas pós-materialistas (Inglehart, 1997) ou o fato de a Declaração Universal dos Direitos Humanos e diversas legislações nacionais e internacionais condenarem todas as formas de preconceito e discriminação, são fatores que têm contribuído para o progressivo estabelecimento de uma norma social anti-preconceito (Alonso, 2007; Castillo, Rodríguez, Torres, Pérez, \& Martel, 2003; Pereira, Monteiro, \& Camino, 2009). A progressiva influência desta norma tem levado a que algumas pessoas tendam a evitar expressar de forma aberta e declarada atitudes negativas contra lésbicas e gays (Castillo et al., 2003; Pereira et al., 2009). Consistentemente, um inquérito sobre diversidade sexual e homofobia realizado no Brasil (Venturi, 2009) revelou que $92 \%$ das pessoas entrevistadas acreditavam que existia preconceito contra pessoas GLBT (gays, lésbicas, bissexuais e transgêneros). No entanto, quando perguntado se eram preconceituosas, apenas entre $26 \%$ a $29 \%$ admitiram ter preconceito contra estas pessoas. Assim, tal como sucedeu com outros grupos discriminados, o preconceito contra lésbicas e gays tem adotado um caráter mais dissimulado ou sutil (para uma revisão das abordagens psicológicas do preconceito contra pessoas não heterossexuais, ver Gato et al., 2011). Desta forma, como salientaram Herek e McLemore (2013), a mensuração de aspectos mais contemporâneos do preconceito sexual apresenta principalmente vantagens em contextos nos quais os respondentes estão altamente motivados para se apresentar a si próprios como não preconceituosos, como por exemplo os estudantes universitários (Gato, Fontaine, \& Carneiro, 2012; Morrison \& Morrison, 2002; Rye \& Meaney, 2010).

Tal como qualquer outra forma de preconceito, o preconceito contra lésbicas e gays está associado a determinadas características psicossociais dos indivíduos. Nesta medida, a investi- 
gação tem demonstrado, por exemplo, que homens e mulheres manifestam níveis diferentes de preconceito face a esta população (D'Augelli \& Hershberger, 1995; Evans, 2001; Iraklis, 2010; Kite \& Whitley, 1996).

Foi objetivo deste trabalho analisar duas expressões do preconceito contra lésbicas e gays, em duas amostras de estudantes universitários portugueses e brasileiros; averiguou-se ainda a influência do gênero na expressão das mesmas. Antes de se apresentarem e discutirem os resultados do estudo empírico realizado, descreve-se brevemente o contexto português e brasileiro no que diz respeito ao preconceito e discriminação contra as pessoas GLBT; distinguem-se as duas expressões do preconceito analisadas neste trabalho; finalmente, estabelece-se uma relação entre o preconceito contra lésbicas e gays e o gênero.

\section{Preconceito em Português}

Em Portugal, na sequência de reivindicações das associações de defesa dos direitos das pessoas GLBT, tem-se assistido, desde o início do século XXI, a importantes alterações legislativas no que concerne aos direitos civis das minorias sexuais (Carneiro \& Menezes, 2007; Vale de Almeida, 2010). Por exemplo, em 2004, a orientação sexual foi incluída no artigo 13 da Constituição Portuguesa (Princípio da Igualdade; Constituição Portuguesa, 2005); em 2010 o acesso ao casamento civil foi garantido a casais do mesmo sexo. Não obstante estas mudanças, continuam-se a registrar níveis mais elevados de preconceito e discriminação contra lésbicas e gays em Portugal, do que na maior parte dos seus países congêneres da Europa ocidental (Comissão Europeia, 2012; European Union Agency for Fundamental Rights [EUFRA], 2013; Jackle \& Wenzelburger, 2014; Stulhofer \& Rimac, 2009; Takács \& Szalma, 2013; van den Akker et al., 2013). Assim, em 2012, 55\% dos Portugueses acreditavam que a discriminação com base na orientação sexual era muito frequente no seu país (média na União Europeia de 46\%). Um inquérito realizado em Portugal a 972 pessoas com orientações e identidades de gênero não normativas (Nogueira \& Oliveira, 2010) revelou efetivamente que estas expressavam uma percepção elevada de discriminação, quer por parte da população geral, quer em setores específicos como a Justiça, a Educação, a Segurança Social e a Saúde.

Dados de um inquérito europeu realizado a pessoas GLBT (EUFRA, 2013) indicam que apenas $4 \%$ dos respondentes portugueses consideraram que no seu país eram muito frequentes as medidas positivas para promover o respeito pelos seus direitos humanos. No que diz respeito à sua vida cotidiana, $51 \%$ dos respondentes sentiram-se discriminados ou assediados com base na sua orientação sexual nos 12 meses anteriores; $59 \%$ afirmaram evitar dar as mãos a um parceiro do mesmo sexo em público com receio de ser atacados, ameaçados ou assediados.

No Brasil, embora a Constituição Federal de 1988 não considere a orientação sexual entre as formas de discriminação, algumas constituições estaduais e legislações municipais já contemplam explicitamente esse tipo de discriminação (Conselho Nacional de Combate à Discriminação [CNCD] \& Ministério da Saúde, 2004; Nardi, 2013). Algumas ações judiciais movidas por grupos de ativistas GLBT têm também redundado em mudanças legislativas como, por exemplo, a extensão de benefícios de pensão por morte e auxílio-reclusão aos casais de pessoas do mesmo sexo e a abertura de precedentes jurisprudenciais relacionados com direitos de parentalidade de pessoas homossexuais (CNCD \& Ministério da Saúde, 2004; Nardi, 2013). Saliente-se, no entanto, que os crimes de ódio contra pessoas GLBT e mais especialmente contra travestis e transgêneros - são um dos problemas com que o Brasil se depara (CNCD \& Ministério da Saúde, 2004). Para além destas situações extremas, Carrara, Ramos, e Caetano (2002, citados pelo CNCD \& Ministério da Saúde, 2004) mostraram a existência de outras formas de preconceito e discriminação. Assim, este estudo realizado no Rio de Janeiro, envolvendo 416 pessoas GLT (Carrara et al., 2002, citados pelo CNCD \& Ministério da Saúde, 2004) revelou, entre outros, que 56,3\% dos inquiridos declararam já haver passado pela experiência de ouvir xingamentos, ofensas verbais e ameaças relacionadas à homossexualida- 
de. Além disso, devido a sua orientação sexual ou identidade de gênero, $58,5 \%$ declararam já haver experimentado discriminação ou humilhação tais como impedimento de ingresso em estabelecimentos comerciais, expulsão de casa, mau tratamento por parte de servidores públicos, colegas, amigos e familiares, chacotas, problemas na escola, no trabalho ou no bairro. Consistentemente, os resultados da pesquisa "Juventudes e sexualidade" (Castro, Abramovay, \& Silva, 2004), realizada em 14 capitais brasileiras, apontam para níveis elevados de preconceito contra as pessoas GLBT. Por exemplo, $25 \%$ dos inquiridos não gostariam de ter um colega de classe homossexual (resposta maioritariamente emitida por estudantes do sexo masculino).

Embora a violência e os crimes de ódio contra pessoas GLBT tenham inegavelmente uma expressão mais elevada no Brasil do que em Portugal, é verdade que, a nível atitudinal, o preconceito contra lésbicas e gays parece estar presente de forma semelhante nestes dois países.

\section{Algumas Nuances do Preconceito Contra Lésbicas e Gays}

Dada a já referida influência da norma social anti-preconceito, importa analisar de forma mais aprofundada que tipos de atitudes negativas face a lésbicas e a gays coexistem atualmente nas sociedades contemporâneas, desde as mais tradicionais até às mais modernas. No que diz respeito às primeiras, são ainda visíveis diversas manifestações de condenação moral e patologização da homossexualidade. Por exemplo, o Instituto Português de Sangue exclui explicitamente os homens que têm sexo com homens da doação voluntária de sangue, deliberação justificada com a alegação de que os gays são sexualmente mais "promíscuos" do que os heterossexuais (Carneiro, 2006; Veludo, 2009). Apesar de desde 1973 a homossexualidade não constar da lista de doenças mentais da Associação Americana de Psiquiatria, 36 anos mais tarde o presidente do Colégio de Psiquiatria da Ordem dos Médicos Portugueses ainda distinguia entre homossexualidade primária (adquirida biologicamente) e secundária (adquirida socialmente), declarando que a última pode ser objeto de tratamento psiquiátrico (Sanches, 2009).

No Brasil, a homossexualidade foi tratada pela Medicina e pela Psicologia como patologia até muito recentemente (Scardua \& Souza, 2006). Efetivamente, só em 1999 foi promulgada uma resolução do Conselho Federal de Psicologia que estabelece aos psicólogos normas de atuação em relação às orientações sexuais, em que a homossexualidade não é considerada doença, nem distúrbio, não podendo estes trabalhar em propostas de tratamento e cura da mesma (Lacerda, Pereira, \& Camino, 2002).

Sendo ainda possível encontrar visões abertamente negativas e patologizadoras da homossexualidade, também é verdade que, como já foi referido, o preconceito contra as pessoas não heterossexuais tornou-se mais sutil, isto é, assumiu formas menos evidentes. Efetivamente, estas transformações encontram um paralelo, quer no domínio dos novos preconceitos raciais e étnicos (Gaertner \& Dovidio, 2000; McConahay, 1986; Meertens \& Pettigrew, 1999), quer no domínio do preconceito de gênero contemporâneo (Glick \& Fiske, 2001; Swim, Aikin, Hall, \& Hunter, 1995; Tougas, Brown, Beaton, \& Joly, 1995). No entanto, o preconceito moderno contra lésbicas e gays não encontra necessariamente um paralelo nestes novos preconceitos raciais e sexistas (Gato et al., 2011). De acordo com Hegarty (2006), mais do que receio da discriminação positiva, de competição econômica ou de diferenças culturais inconciliáveis, patentes, por exemplo, no racismo moderno (McConahay, 1986), as expressões mais contemporâneas do preconceito contra as pessoas GLBT radicam essencialmente no desconforto com a sua visibilidade. Esta visibilidade é acentuada pelo crescente acesso das pessoas GLBT a instituições tradicionalmente associadas à heterossexualidade como, por exemplo, o casamento e a parentalidade (Gato et al., 2011). Assim, no caso da orientação sexual, o que está em jogo no que diz respeito ao preconceito sexual moderno relaciona-se sobretudo com a influência do heterossexismo, ideologia que valoriza a heterossexualidade como mais natural que e/ou superior à homossexualidade (Morin, 1977). 


\section{Gênero e Preconceito Contra Lésbicas e Gays}

O gênero tem sido identificado como um dos preditores mais confiáveis do preconceito contra lésbicas e gays (para uma meta-análise ver Kite $\&$ Whitley, 1996), sendo as atitudes dos homens mais homofóbicas do que as das mulheres. Este padrão foi também observado em amostras de estudantes universitários (D'Augelli \& Hershberger, 1995; Evans, 2001; Iraklis, 2010). Este fenômeno pode ser melhor compreendido a partir de uma articulação de diversos contributos teóricos, provenientes de várias áreas das ciências sociais. São particularmente úteis no campo sociológico, as perspectivas do doing gender (West \& Zimmerman, 1987, 2002) e da masculinidade hegemônica (Connell, 1987, 1995) e, no campo da psicologia social, os estudos acerca dos estereótipos de gênero (Eagly, Wood, \& Diekman, 2000; Fiske, Cuddy, Glick, \& Xu, 2002; Pratto \& Walker, 2004; Williams \& Best, 1990).

West e Zimmerman $(1987,2002)$ argumentaram que o gênero não é algo que somos, mas sim algo que fazemos e que é construído em interacção. Fazer gênero é, nesta perspectiva, agir com a possibilidade de se ser julgado segundo com a categoria sexual a que se pertence ou aparenta pertencer, isto é, de "prestar contas" a essa categoria sexual. O gênero é, assim, socialmente construído e estabelecido nas interações cotidianas, à luz de concepções normativas da masculinidade e da feminilidade. Em geral, estas concepções ou estereótipos descrevem as mulheres como mais comunais e expressivas e os homens como mais instrumentais (Eagly et al., 2000). Contudo, para além desta dimensão horizontal de diferença, existe uma dimensão hierárquica de desigualdade, sendo aos homens atribuído um maior poder (Pratto \& Walker, 2004). Williams e Best (1990) mostraram que, atribuindo a cada sexo aptidões específicas, os estereótipos de gênero associam simultaneamente um estatuto superior e maior competência aos homens, particularmente no que diz respeito às áreas socialmente valorizadas da racionalidade e da instrumentalidade. Da mesma forma, Fiske et al. (2002) demonstraram que as mulheres são, em geral, vistas como menos competentes, mas mais "amistosas" e mais proficientes em tarefas comunais (por sua vez menos valorizadas).

Connell $(1987,1995)$ também enfatiza que a masculinidade e a feminilidade são conceitos relacionais. Por esta razão, ao longo das suas vidas, a generalidade dos homens procurar afirmar a sua masculinidade provando que não são mulheres. Nas palavras de Kimmel, a "noção de anti-feminilidade está no cerne das construções históricas e contemporâneas da masculinidade, de tal forma que a masculinidade se define mais pelo que não se é do que pelo que se é" (1994, p. 119). Postulando os estereótipos acerca das lésbicas e dos gays que estes possuem os traços do sexo "oposto" (Kite \& Deaux, 1987), a homossexualidade é geralmente associada um comportamento de gênero invertido. Adicionalmente, dado que a masculinidade hegemônica é definida como exclusivamente heterossexual (Connell, 1987, 1995; Kimmel, 1994), a homossexualidade masculina é vista como uma contradição e uma expressão de feminilidade. De forma consistente, a homossexualidade masculina é mais repudiada do que a homossexualidade feminina, particularmente pelos homens heterossexuais (Kite \& Whitley, 1996). A homofobia é assim essencial para a rejeição da feminilidade e afirmação da masculinidade heterossexual (Badinter, 1997; Connell, 1987, 1995; Herek, 1993). Como referiu Connell (1987, p. 186), “o desprezo pela homossexualidade e pelos homens homossexuais... faz parte do pacote ideológico da masculinidade hegemônica".

No estudo empírico que se apresenta em seguida, serão avaliadas, numa amostra de estudantes brasileiros e portugueses, duas manifestações do preconceito contra lésbicas e gays: uma de caráter mais tradicional e outra de caráter mais contemporâneo. Uma vez que estas atitudes não são independentes do gênero, será também explorada a relação com essa variável. Foram, assim, testadas as seguintes hipóteses:

Hipótese 1: Não se antecipam diferenças entre Brasil e Portugal nas duas expressões do preconceito.

Hipótese 2: Os participantes evidenciarão níveis mais elevados da expressão mais contemporânea do preconceito do que da sua expressão tradicional. 
Hipótese 3: Os participantes do sexo masculino apresentarão níveis mais elevados de preconceito do que as suas congêneres do sexo feminino.

\section{Método}

\section{Participantes}

A amostra, não probabilística, é constituída por 844 estudantes provenientes de univer- sidades públicas de Portugal (Universidade do Porto, Universidade de Lisboa, Universidade da Beira Interior e Universidade do Algarve) e do Brasil (Universidade Federal de Uberlândia). A idade variou de 17 a 60 anos, com uma média de 22 anos $(D P=5,03)$. Como se pode constatar através da Tabela 1 , as amostras foram equilibradas em termos do curso e do gênero dos participantes.

Tabela 1

Caracterização da Amostra

\begin{tabular}{lccccc}
\hline País & \multicolumn{2}{c}{ Brasil (B) } & \multicolumn{2}{c}{ Portugal (P) } \\
\hline Curso/Gênero & Mulheres & Homens & Mulheres & Homens & Total \\
\hline Medicina & 62 & 37 & 76 & 28 & 203 \\
& $(63 \%)$ & $(37 \%)$ & $(73 \%)$ & $(27 \%)$ & $(24 \%)$ \\
Direito & 76 & 48 & 49 & 18 & 191 \\
& $(61 \%)$ & $(39 \%)$ & $(73 \%)$ & $(27 \%)$ & $(23 \%)$ \\
Ciências Sociais (B)/Sociologia (P) & 27 & 10 & 27 & 12 & 76 \\
& $(73 \%)$ & $(27 \%)$ & $(70 \%)$ & $(30 \%)$ & $(9 \%)$ \\
Engenharia Mecânica & 11 & 93 & 15 & 126 & 245 \\
& $(11 \%)$ & $(89 \%)$ & $(11 \%)$ & $(89 \%)$ & $(29 \%)$ \\
Pedagogia (B)/Ciências da Educação (P) & 52 & 4 & 59 & 14 & 129 \\
& $(93 \%)$ & $(7 \%)$ & $(81 \%)$ & $(19 \%)$ & $(15 \%)$ \\
\hline \multirow{2}{*}{ Total } & 228 & 192 & 226 & 198 & 844 \\
& $(54 \%)$ & $(46 \%)$ & $(53 \%)$ & $(47 \%)$ & $(100 \%)$ \\
\hline
\end{tabular}

\section{Instrumento}

A Escala Multidimensional de Atitudes Face a Lésbicas e a Gays (EMAFLG) foi construída e validada em Portugal (Gato et al., 2012) e é composta por três dimensões atitudinais negativas, duas de caráter mais tradicional (homopatologização e rejeição da proximidade) e uma de caráter mais contemporâneo (heterossexismo moderno), e por uma atitude positiva (Suporte). O instrumento foi também validado no Brasil (Gato, Fontaine, \& Leme, 2014), sendo, portanto, possível a sua utilização em amostras dos dois países. Neste estudo foram utilizadas como variáveis dependentes duas dimensões negativas da EMAFLG: a homopatologização e o heterossexismo moderno.
Os cinco itens que compõem a sub-escala homopatologização dizem respeito a juízos morais e patologizadores acerca da homossexualidade (por exemplo, "A homossexualidade é uma perturbação psicológica"). O heterossexismo moderno é composto por sete itens relacionados com manifestações contemporâneas do preconceito contra lésbicas e gays. Estas referem-se ao desempenho por estas pessoas de papéis tradicionalmente associados à heterossexualidade, como a conjugalidade e a parentalidade. Incluem-se ainda neste tipo de preconceito posicionamentos negativos face à visibilidade ou expressão da identidade lésbica e gay e à ênfase excessiva que lésbicas e gays supostamente colocam na sua sexualidade (por exemplo, "Ser criado num lar 
homossexual é bastante diferente de ser criado num lar heterossexual" ou "Celebrações como o 'dia do orgulho gay' são ridículas porque assumem que a orientação sexual deve constituir um motivo de orgulho").

Os participantes exprimiram a sua opinião acerca dos itens utilizando uma escala tipo Li- kert de 1 (discordo completamente) a 6 (concordo completamente). Os índices de consistência interna das duas dimensões apresentaram valores bastante razoáveis, quer para a amostra total, quer para cada um dos países (ver Tabela 2). Além da EMAFLG, os participantes responderam ainda a um conjunto de questões sociodemográficas.

Tabela 2

Consistência Interna (Alfa de Cronbach) da Homopatologização e do Heterossexismo Moderno em Função do País e da Amostra Total

\begin{tabular}{lccc}
\hline & Brasil & Portugal & Amostra Total \\
\hline Homopatologização & 0,84 & 0,86 & 0,85 \\
Heterossexismo moderno & 0,79 & 0,81 & 0,80 \\
\hline
\end{tabular}

\section{Procedimento}

Após ter sido obtida autorização das respectivas instituições, os questionários foram coletivamente administrados no período disponibilizado para o efeito. Antes do preenchimento, foi fornecida informação acerca da natureza voluntária da participação, bem como da confidencialidade e anonimato das respostas. A administração ocorreu em ambiente de sala de aula.

\section{Análise de Dados}

Para averiguar diferenças nas variáveis em função do país e do gênero recorreu-se ao teste $t$ de Student. Para verificar se os níveis de homopatologização diferiam dos níveis de heterossexismo moderno foram realizados testes $t$ de Student para amostras emparelhadas.

\section{Resultados e Discussão}

O objetivo principal desse estudo foi o de analisar duas formas de preconceito contra lésbicas e gays, em duas amostras de estudantes universitários de Portugal e do Brasil, averiguando também a influência do gênero na expressão das mesmas. Todas as hipóteses encontraram confirmação. Assim, não se verificaram diferenças em função do país nos dois tipos de preconceito (hipótese 1). Adicionalmente, os participantes de Portugal e do Brasil evidenciaram níveis signi- ficativamente mais elevados de heterossexismo moderno do que de homopatologização (hipótese 2). Quanto ao gênero, os homens evidenciaram níveis mais elevados das duas formas de preconceito (hipótese 3). Seguidamente, apresentam-se e discutem-se esses resultados em maior detalhe, reflete-se acerca das limitações e contributos do estudo, retirando-se ainda algumas implicações do mesmo.

Como se pode constatar na Tabela 3 não se verificaram diferenças em função do país nas duas variáveis, resultado que encontra aliás eco nos dados provenientes do World Values Survey (Jackle \& Wenzelburger, 2014), em que a média das respostas ao indicador de atitudes perante a homossexualidade, foi bastante semelhante nos dois países. Para além de uma certa proximidade cultural entre Portugal e o Brasil, há que ter em conta na explicação deste resultado o fato de se estar em presença de duas amostras bastante homogêneas no que às suas características diz respeito.

Os participantes portugueses e brasileiros evidenciaram níveis significativamente mais elevados de heterossexismo moderno do que de homopatologização (diferenças assinaladas através das letras $a$ e $b$ na Tabela 3). Tratando-se de respondentes com um elevado nível de educação, os estudantes portugueses e brasileiros poderão ter interiorizado, de forma semelhante, 
Tabela 3

Diferenças em Função do País

\begin{tabular}{lccccccc}
\hline & \multicolumn{2}{c}{ Brasil } & \multicolumn{2}{c}{ Portugal } \\
\hline Variável & $M$ & $D P$ & $M$ & $D P$ & $t$ & $g l$ & $p$ \\
\hline Homopatologização & $2,05^{\mathrm{a}}$ & 1,10 & $1,92^{\mathrm{b}}$ & 1,03 & 1,79 & 815,06 & 0,08 \\
Heterossexismo moderno & $3,42^{\mathrm{a}}$ & 0,81 & $3,52^{\mathrm{b}}$ & 0,71 & 1,73 & 829 & 0,07 \\
\hline
\end{tabular}

a Valores médios de Homopatologização e Heterossexismo, para o Brasil, $t(412)=37,45, p<0,001$ e ${ }^{\mathrm{b}}$ para Portugal, $t(405)=$ $39,96, p<0,001$.

a norma social que impede expressões flagrantemente abertas do preconceito contra lésbicas e gays (Alonso, 2007; Castillo et al., 2003; Herek \& McLemore, 2013; Pereira et al., 2009). Nesta medida, tal como outros congêneres seus, tenderam a subscrever formas mais veladas de preconceito (Gato et al., 2012; Morrison \& Morrison, 2002; Rye \& Meaney, 2010). Tal como aconteceu com outros grupos discriminados (Gaertner \& Dovidio, 2000; Glick \& Fiske, 2001; McConahay, 1986; Meertens \& Pettigrew, 1999; Swim et al., 1995; Tougas et al., 1995), o preconceito contra lésbicas e gays assumiu neste estudo uma expressão mais dissimulada. Assim, o preconceito observado expressou-se menos através de uma atitude patologizadora (Carneiro, 2006; Lacerda et al., 2002; Sanches, 2009; Scardua \& Souza, 2006; Veludo, 2009) do que através de uma recusa da visibilidade de lésbicas e gays e uma maior valorização da heterossexualidade (Hegarty, 2006; Morin, 1977), particularmente no que diz respeito à afirmação de papéis sociais a ela tradicionalmente associados, como a conjugalidade e a parentalidade (Gato et al., 2011). Pelos motivos acima referenciados, a composição estudantil da amostra poderá explicar parcialmente esta maior adesão a um preconceito moderno do que a um preconceito tradicional. Nesta medida, os resultados não deverão ser generalizados para outros setores da população. Em suma, não obstante alguns avanços, principalmente no que diz respeito aos direitos civis das minorias sexuais (CNCD \& Ministério da Saúde, 2004; Constituição Portuguesa, 2005; Inglehart, 1997; Nardi, 2013; Vale de Almeida, 2010), o preconceito contra lésbicas e gays continua a fazer-se sentir em Portugal e no Brasil (Castro et al., 2004; Comissão Europeia, 2012; CNCD \& Ministério da Saúde, 2004; EUFRA, 2013; Nogueira \& Oliveira, 2010; Venturi, 2009), particularmente no que diz respeito à sua expressão mais moderna.

No que concerne ao gênero, como se pode verificar na Tabela 4, observaram-se diferenças entre homens e mulheres, quer na homopatologização, quer no heterossexismo moderno.

Tabela 4

Diferenças em Função do Gênero

\begin{tabular}{|c|c|c|c|c|c|c|c|}
\hline \multirow[b]{2}{*}{ Variável } & \multicolumn{2}{|c|}{ Mulheres } & \multicolumn{2}{|c|}{ Homens } & \multirow[b]{2}{*}{$t$} & \multirow[b]{2}{*}{$g l$} & \multirow[b]{2}{*}{$p$} \\
\hline & $M$ & $D P$ & $M$ & $D P$ & & & \\
\hline Homopatologização & 1,78 & 0,96 & 2,24 & 1,12 & 6,53 & 795,70 & $<0,001$ \\
\hline Heterossexismo moderno & 3,31 & 0,73 & 3,65 & 0,76 & 6,39 & 829 & $<0,001$ \\
\hline
\end{tabular}

Estes resultados estão de acordo com os estudos que indicam que o gênero é um dos preditores mais robustos do preconceito contra lésbicas e gays (Kite \& Whitley, 1996), inclu- sive em pesquisas conduzidas junto de amostras de estudantes universitários (D'Augelli \& Hershberger, 1995; Evans, 2001; Iraklis, 2010). Verifica-se assim que os homens são mais infle- 
xíveis do que as mulheres no que diz respeito às normas de gênero, julgando de forma mais severa aqueles que consideram desviar-se dessas mesmas normas (Badinter, 1997; Connell, 1987, 1995; Eagly et al., 2000; Fiske et al., 2002; Herek, 1993; Kimmel, 1994; Kite \& Deaux, 1987; Kite \& Whitley, 1996; Pratto \& Walker, 2004; West \& Zimmerman, 1987, 2002; Williams \& Best, 1990). Adicionalmente, os resultados deste estudo evidenciam que o gênero influencia de igual forma expressões tradicionais modernas do preconceito contra lésbicas e gays.

Algumas limitações desse estudo devem ser tidas em consideração. Em primeiro lugar, embora as amostras tenham sido emparelhadas em função do gênero e do curso de pertença, são constituídas exclusivamente por estudantes universitários, não sendo por isso representativas da população geral. Em segundo lugar, a orientação sexual dos participantes não era conhecida. Contudo, uma vez que as pessoas não heterossexuais detêm, com maior probabilidade, atitudes menos estereotipadas e hostis perante os seus congêneres, a presença de participantes lésbicas e gays pode ter reduzido a amplitude das diferenças observadas nas atitudes mensuradas. Deve, no entanto, ser notado que esta limitação aumenta a probabilidade de ocorrência do erro de tipo-II, não invalidando os resultados.

Para além destes limites, a presente investigação apresentou também alguns contributos. Em primeiro lugar, tendo sido avaliados dois tipos de preconceito contra lésbicas e gays, o conhecimento sobre esta temática nos dois países, anteriormente adquirido apenas através de indicadores sociológicos (Jackle \& Wenzelburger, 2014; Stulhofer \& Rimac, 2009; Takács \& Szalma, 2013; van den Akker et al., 2013), foi aprofundado. Em segundo lugar, recorreu-se a um instrumento cuja estrutura fatorial foi validada em Portugal (Gato et al., 2012) e no Brasil (Gato et al., 2014), o que fornece garantia de se terem avaliado os mesmos constructos nos dois países.

Finalmente, podem ser retiradas deste estudo algumas implicações em termos de intervenção. Dadas as amostras estudadas, estas dizem sobretudo respeito ao contexto universitário.
Considerando o seu papel, quer em termos de formação científica, quer de formação pessoal e de cidadania dos/as estudantes, este contexto parece propício para se agir sobre a desinformação e o preconceito que subsistem face a lésbicas e a gays (Kim, D'Andrea, Gaughen, \& Sahu, 1998). Com base nos resultados obtidos, os agentes educativos e psicossociais, particularmente aqueles que operam no espaço universitário, devem ser sensíveis, quer a expressões mais modernas do preconceito, quer à maior incidência deste na população masculina.

\section{Referências}

Allport, G. (1997). The nature of prejudice. Reading, MA: Addison-Wesley. (Original work published 1954)

Alonso, F. M. (2007). Prejuicio y estigma: efectos y posibles soluciones. In J. F. M. Morales, M. C. Moya, E. Gaviria, \& I. Cuadrado (Eds.), Psicología social (3. ed., pp. 619-640). Madrid, España: McGraw-Hill.

Badinter, E. (1997). XY: A identidade masculina (3. ed.). Porto, Portugal: ASA.

Carneiro, N. S. (2006). Ser, pertencer e participar: Construção da identidade homossexual, redes de apoio e participação comunitária (Tese de doutorado não publicada, Faculdade de Psicologia e de Ciências da Educação, Universidade do Porto, Portugal).

Carneiro, N. S., \& Menezes, I. (2007). From an oppressed citizenship to affirmative identities: Lesbian and gay political participation in Portugal. Journal of Homosexuality, 53(3), 65-82. doi:10.1300/J082v53n03 05

Castillo, M. N. Q., Rodríguez, V. B., Torres, R. R., Pérez, A. R., \& Martel, E. C. (2003). La medida de homofobia manifiesta y sutil. Psicothema, 15(2), 197-204.

Castro, M. G., Abramovay, M., \& Silva, L. B. (2004). Juventudes e sexualidades. Brasília, DF: Organização das Nações Unidas para a Educação, a Ciência e a Cultura.

Comissão Europeia. (2012). Special Eurobarometer 393: Discrimination in the EU in 2012 (Report Wave EB77.4 - TNS Opinion \& Social). Retrieved from http://ec.europa.eu/public_opinion/archives/ebs/ebs_393_en.pdf 
Connell, R. W. (1987). Gender and power: Society, the person and sexual politics. Stanford, CA: Stanford University Press.

Connell, R. W. (1995). Masculinities. Cambridge, MA: Polity Press.

Conselho Nacional de Combate à Discriminação, \& Ministério da Saúde. (2004). Brasil sem homofobia: Programa de combate à violência e à discriminação contra GLTB e promoção da cidadania homossexual. Brasília, DF: Ministério da Saúde.

Constituição Portuguesa. (2005). Princípios fundamentais. Recuperado em http://www.parlamento.pt/Legislacao/Paginas/ConstituicaoRepublicaPortuguesa.aspx

D’Augelli, A. R., \& Hershberger, S. L. (1995). A multiyear analysis of changes in AIDS: Concerns and homophobia on a university campus. Journal of American College Health, 44, 3-10.

Dovidio, J. F., \& Gaertner, S. L. (2010). Intergroup bias. In S. T. Fiske, D. T. Gilbert, \& G. Lindzey (Eds.), Handbook of Social Psychology (pp. 1084-1121). Hoboken, NJ: Wiley.

Duckitt, J. H. (1992). The social psychology of prejudice. New York: Praeger.

Eagly, A. H., Wood, W., \& Diekman, A. B. (2000). Social role theory of sex differences and similarities: A current appraisal. In T. Eckes \& H. M. Trautner (Eds.), The developmental social psychology of gender (pp. 123-174). Mahwah, NJ: Lawrence Erlbaum.

European Union Agency for Fundamental Rights. (2013). EU LGBT survey. European Union lesbian, gay, bisexual and transgender survey. Results at a glance. Retrieved from http://fra. europa.eu/sites/default/files/eu-lgbt-survey-results-at-a-glance_en.pdf

Evans, N. J. (2001). The experiences of lesbian, gay and bisexual youths in university communities. In A. R. D’Augelli \& C. J. Patterson (Eds.), Lesbian, gay, and bisexual identities and youth: Psychological perspectives (pp. 181-198). New York: Oxford University Press.

Fiske, S. T. (1998). Stereotyping, prejudice, and discrimination. In D. T. Gilbert, S. T. Fiske, \& G. Lindzey (Eds.), Handbook of social psychology ( $4^{\text {th }}$ ed., Vol. 2, pp. 357-411). Boston, MA: McGraw-Hill.
Fiske, S. T., Cuddy, A. J., Glick, P., \& Xu, J. (2002). A model of (often mixed) stereotype content: Competence and warmth respectively follow from perceived status and competence. Journal of Personality and Social Psychology, 82, 878902. doi:10.1037//0022-3514.82.6.878

Gaertner, S. L., \& Dovidio, J. F. (Eds.). (2000). Reducing intergroup bias: The common ingroup identity model. Hove, UK: Psychology Press.

Gato, J., Carneiro, N. S., \& Fontaine, A. M. (2011). Contributo para uma revisitação histórica e crítica do preconceito contra as pessoas não heterossexuais. Crítica e Sociedade: Revista de Cultura Politica, 1(1), 139-167.

Gato, J., Fontaine, A. M., \& Carneiro, N. S. (2012). Escala Multidimensional de Atitudes Face a Lésbicas e a Gays: Construção e validação preliminar. Paidéia (Ribeirão Preto), 22(51), 11-20. doi:10.1590/S0103-863X2012000100003

Gato, J., Fontaine, A. M., \& Leme, V. B. R. (2014). Validação e adaptação transcultural da Escala Multidimensional de Atitudes Face a Lésbicas e a Gays. Psicologia: Reflexão e Crítica, 27(2), 257-271. doi:10.1590/1678-7153.201427206

Glick, P., \& Fiske, S. T. (2001). Ambivalent sexism. In M. P. Zanna (Ed.), Advances in experimental social psychology (Vol. 33, pp. 115-188). San Diego, CA: Academic Press.

Hegarty, P. (2006). Where's the sex in sexual prejudice. Lesbian \& Gay Psychology Review, 7(3), 264-275.

Herek, G. M. (1993). On heterosexual masculinity: Some psychical consequences of the social construction of gender and sexuality. In L. D. Garnets \& D. C. Kimmel (Eds.), Psychological perspectives on lesbian and gaymale experiences (pp. 316-330). New York: Columbia University Press.

Herek, G. M. (2009). Sexual prejudice. In T. Nelson (Ed.), Handbook of prejudice, stereotyping, and discrimination (pp. 439-465). New York: Psychology Press.

Herek, G. M., \&McLemore, K.A.(2013). Sexualprejudice. Annual Review of Psychology, 64, 309-333. doi:10.1146/annurev-psych-113011-143826

Inglehart, R. (1997). Modernization and postmodernization: Cultural, economic, and political change in 43 societies. Princeton, NJ: Princeton University Press. 
Iraklis, G. (2010). Predictors of Greek students - attitudes towards lesbians and gay men. Psychology \& Sexuality, 1(2), 170-179. doi:10.1080/194198 99.2010 .484598

Jackle, S., \& Wenzelburger, G. (2014). Religion, religiosity and the attitudes towards homosexuality - A multilevel analysis of 79 countries. Journal of Homosexuality, 62(2), 207-241. doi:10.1080/ 00918369.2014 .969071

Kim, B. S. K., D’Andrea, M. J., Gaughen, K. J. S., \& Sahu, P. K. (1998). A multicultural study of university students' knowledge of and attitudes toward homosexuality. Journal of Humanistic Education and Development, 36(3), 171-182.

Kimmel, M. S. (1994). Masculinity as homophobia: Fear, shame, and silence in the construction of gender identity. In H. Brod \& M. Kaufman (Eds.), Theorizing Masculinities (pp. 119-141). Thousand Oaks, CA: Sage.

Kite, M. E., \& Deaux, K. (1987). Gender belief systems: Homosexuality and implicit inversion theory. Psychology of Women Quarterly, 11, 83-96. doi:10.1111/j.1471-6402.1987.tb00776.x

Kite, M. E., \& Whitley, B. E., Jr. (1996). Sex differences in attitudes toward homosexual persons, behaviors, and civil rights: A meta-analysis. Personality and Social Psychology Bulletin, 22, 336-353. doi:10.1177/0146167296224002

Lacerda, M., Pereira, C., \& Camino, L. (2002). Um estudo sobre as formas de preconceito contra homossexuais na perspectiva das representações sociais. Psicologia: Reflexão e Crítica, 15(1), 165 178. doi:10.1590/S0102-79722002000100018

McConahay, J. B. (1986). Modern racism, ambivalence and the modern racism scale. In J. F. Dovidio \& S. L. Gaertner (Eds.), Prejudice, discrimination and racism (pp. 91-124). New York: Academic Press.

Meertens, R., \& Pettigrew, T. F. (1999). Será o racismo subtil mesmo racismo? In J. Vala (Ed.), Novos racismos: Perspectivas comparadas (pp. 11-29). Oeiras, Portugal: Celta.

Morin, S. F. (1977). Heterosexual bias in psychological research on lesbianism and male homosexuality. American Psychologist, 32(8), 629-637. doi:10.1037/0003066X.32.8.629

Morrison, M. A., \& Morrison, T. G. (2002). Development and validation of a scale measuring modern prejudice toward gay men and lesbian women. Journal of Homosexuality, 43(2), 1537. doi:10.1300/J082v43n02_02

Nardi, H. C. (2013). Relações de gênero e diversidade sexual: Compreeendendo o contexto sociopolítico contemporâneo. In H. C. Nardi, R. S. Silveira, \& P. S. Machado (Eds.), Diversidade sexual, relações de gênero e políticas públicas (pp. 1531). Porto Alegre, RS: Sulina.

Nogueira, C., \& Oliveira, J. M. (2010). Estudo sobre a discriminação em função da orientação sexual e da identidade de género. Lisboa, Portugal: Comissão para a Cidadania e a Igualdade de Género.

Pereira, A., Monteiro, M. B., \& Camino, L. (2009). Social norms and prejudice against homosexuals. The Spanish Journal of Psychology, 12(2), 576-584. doi:10.1017/S1138741600001943

Plous, S. (2003). The psychology of prejudice, stereotyping and discrimination: An overview. In S. Plous, Understanding prejudice and discrimination (pp. 3-48). New York: McGraw-Hill.

Pratto, F., \& Walker, A. (2004). The bases of gendered power. In A. H. Eagly, A. E. Beall, \& R. J. Sternberg (Eds.), The psychology of gender ( $2^{\text {nd }}$ ed., pp. 242-268). New York: The Guilford Press.

Quillian, L. (2006). New approaches to understanding racial prejudice and discrimination. Annual Review of Sociology, 32, 299-328.

Rye, B. J., \& Meaney, G. J. (2010). Self-defense, sexism, and etiological beliefs: Predictors of attitudes toward gay and lesbian adoption. Journal of GLBT Family Studies, 6, 1-24. doi:10.1080/15504280903472485

Sanches, A. (2009, 02 maio). Tratamentos para "alterar" orientação sexual não são uma coisa do passado. Jornal Público / P2. Recuperado em http:// pt.scribd.com/doc/15376340/Tratamentos-para-alterar-orientacao-sexual-nao-sao-uma-coisa-do-passado-02052009-Andreia-Sanches

Scardua, A., \& Souza, E. A., Filho. (2006). O debate sobre a homossexualidade mediado por representações sociais: Perspectivas homossexuais e heterossexuais. Psicologia: Reflexão e Crítica, 19(3), 482-490. doi:10.1590/S010279722006000300017

Stulhofer, A., \& Rimac, I. (2009). Determinants of homonegativity in Europe. Journal of Sex Research, 46(1), 24-32. doi:10.1080/00224490802398373 
Swim, J. K., Aikin, K. J., Hall, W. S., \& Hunter, B. A. (1995). Sexism and racism: Old-fashioned and modern prejudices. Journal of Personality and Social Psychology, 68(2), 199-214. doi:10.1037/0022-3514.68.2.199

Takács, J., \& Szalma, Y. (2013). How to measure homophobia in an international comparison. Druzhoslovne Razparave, 29(73), 11-42.

Tougas, F., Brown, R., Beaton, A. M., \& Joly, S. (1995). Neosexism: plus ça change, plus c'est pareil. Personality and Social Psychology Bulletin, 21, 842-849. doi:10.1177/0146167295218007

Vale de Almeida, M. (2010). O contexto LGBT em Portugal. In C. Nogueira \& J. M. Oliveira (Eds.), Estudo sobre a discriminação em função da orientação sexual e da identidade de género (pp. 45-92). Lisboa, Portugal: Comissão para a Cidadania e a Igualdade de Género.

Van den Akker, H., van der Ploeg, R., \& Scheepers, P. (2013). Disapproval of homosexuality: Comparative research on individual and national determinants of disapproval of homosexuality in 20 European countries. International Journal of Public Opinion Research, 25(1), 64-86. doi:10.1093/ijpor/edr058

Veludo F. (2009, 17 jul.). Recusa de dádivas de sangue por homossexuais: Presidente do Instituto Português do Sangue nega discriminação e alega evidências científicas. Jornal Público / P2. Recuperado em http://www.publico.pt/sociedade/ noticia/presidente-do-instituto-portugues-do-sangue-nega-discriminacao-e-alega-evidencias-cientificas-1392233
Venturi, G. (2009). Pesquisa - Intolerância à diversidade sexual. Recuperado em http:// www. fpanramo.org.br/o-que-fazemos/pesquisas-de-opiniao-publica/pesquisas-realizadas/apresentacao-4

Weinberg, G. (1972). Society and the healthy homosexual. New York: St. Martin's Press.

West, C., \& Zimmerman, D. H. (1987). Doing gender. Gender \& Society, 1, 125-151.

West, C., \& Zimmerman, D. H. (2002). Doing gender. In S. Fenstermaker \& C. West (Eds.), Doing gender, doing difference: Inequality, power, and institutional change (pp. 3-23). London: Routledge.

Williams, J. E., \& Best, D. L. (1990). Measuring sex stereotypes: A multinational study. Newbury Park, CA: Sage.
Recebido: 31/02/2012

$1^{a}$ revisão: $31 / 10 / 2014$ Aceite final: $28 / 01 / 2015$ 